\title{
Front-End Module of 18-40 GHz Ultra-Wideband Receiver for Electronic Warfare System
}

\author{
Yuseok Jeon ${ }^{1, *} \cdot$ Sungil Bang ${ }^{2}$
}

\begin{abstract}
In this study, we propose an approach for the design and satisfy the requirements of the fabrication of a small, lightweight, reliable, and stable ultra-wideband receiver for millimeter-wave bands and the contents of the approach. In this paper, we designed and fabricated a stable receiver with having low noise figure, flat gain characteristics, and low noise characteristics, suitable for millimeter-wave bands. The method uses the chip-and-wire process for the assembly and operation of a bare MMIC device. In order to compensate for the mismatch between the components used in the receiver, an amplifier, mixer, multiplier, and filter suitable for wideband frequency characteristics were designed and applied to the receiver. To improve the low frequency and narrow bandwidth of existing products, mathematical modeling of the wideband receiver was performed and based on this spurious signals generated from complex local oscillation signals were designed so as not to affect the RF path. In the ultra-wideband receiver, the gain was between $22.2 \mathrm{~dB}$ and $28.5 \mathrm{~dB}$ at Band $\mathrm{A}$ (input frequency, 18-26 GHz) with a flatness of approximately $6.3 \mathrm{~dB}$, while the gain was between $21.9 \mathrm{~dB}$ and $26.0 \mathrm{~dB}$ at Band B (input frequency, 26-40 GHz) with a flatness of approximately $4.1 \mathrm{~dB}$. The measured value of the noise figure at Band A was $7.92 \mathrm{~dB}$ and the maximum value of noise figure, measured at $\mathrm{Band} B$ was $8.58 \mathrm{~dB}$. The leakage signal of the local oscillator (LO) was $-97.3 \mathrm{dBm}$ and -90 $\mathrm{dBm}$ at the $33 \mathrm{GHz}$ and $44 \mathrm{GHz}$ path, respectively. Measurement was made at the $15 \mathrm{GHz}$ IF output of band A ( $\mathrm{LO}, 33 \mathrm{GHz})$ and the suppression characteristic obtained through the measurement was approximately $30 \mathrm{dBc}$.
\end{abstract}

Key Words: Down Converter, Dual Input, EW System, Front-End, Local Oscillator, Ultra-Wideband Receiver.

\section{INTRODUCTION}

To penetrate enemy air defense networks protected by overlapping weapon systems and successfully carry out missions, combat aircraft should be installed with electronic warfare (EW) weapon systems, which require electronic support (ES) equipment that enables the aircraft to detect and analyze the enemy's electromagnetic wave signals and locate the signal sources precisely [1].

In particular, as the range of frequencies used by various threatening weapon systems such as radar missiles has increased from microwave bands to millimeter wave bands, ultra-wideband receivers $(18-40 \mathrm{GHz})$ are necessary to receive signals that have very high frequencies [2-4]. Therefore, numerous studies on the design of ultra-wideband receivers for millimeter-wave bands suitable for EW systems are underway. However, there are many constraints for the design and fabrication of such wideband receivers $[5,6]$.

As currently existing wideband receivers made of single modules can only receive signals in bands up to $2-18 \mathrm{GHz}$, they are insufficient for high-frequency signals in millimeter-wave bands. In addition, as the local oscillator (LO) necessary for frequency

Manuscript received February 13, 2018 ; Revised April 10, 2018 ; Accepted June 2, 2018. (ID No. 20180213-019J)

${ }^{1}$ Research and Development Department, Broadern Inc., Hwaseong, Korea.

${ }^{2}$ Division of Electronic and Electric Engineering, Dankook University, Yongin, Korea.

"Corresponding Author: Yuseok Jeon (e-mail: halsuida@naver.com)

This is an Open-Access article distributed under the terms of the Creative Commons Attribution Non-Commercial License (http://creativecommons.org/licenses/by-nc/4.0) which permits unrestricted non-commercial use, distribution, and reproduction in any medium, provided the original work is properly cited.

(c) Copyright The Korean Institute of Electromagnetic Engineering and Science. All Rights Reserved. 
conversion is not implemented in their receiver modules [7], but provided separately with converted frequencies from ES system devices, the phase noise characteristics of the entire receivers are dependent on the performance of other devices, and the size of the entire system increases owing to the separate LO module.

Therefore, the dynamic range (minimum detection level) that can be accepted by the signal processing unit is narrowed, limiting the application of the receivers to the front-end terminals of actual EW systems. In addition, sealing structures are also necessary to avoid signal interference due to other devices in the system environment where the receivers are installed $[8,9]$.

In this paper, a design approach is proposed to overcome the disadvantages of the existing methods and satisfy the requirements of the ultra-wideband receiver for millimeter-wave bands.

First, the ultra-wideband input frequency range of 18-40 $\mathrm{GHz}$ were divided into two bands using $18-26 \mathrm{GHz}$ (Band $\mathrm{A}$ ) and $26-40 \mathrm{GHz}$ (Band B) band pass filters and these were applied to the frequency mixer input. The designed frequency spectra were such that there was no secondary harmonic component in each band, thereby preventing spurious signals from appearing in the intermediate frequencies (IF, 4-18 GHz).

After applying the external reference frequency of $1 \mathrm{GHz}$ to the $\mathrm{LO}$ circuit, common $11 \mathrm{GHz}$ signals were generated to implement $33 \mathrm{GHz}$ (signals of three times of $11 \mathrm{GHz}$ ) and 44 $\mathrm{GHz}$ (signals four times of $11 \mathrm{GHz}$ ) LO frequencies in the receiver module so that the phase noise characteristics are not affected by other devices and the complexity of the circuit was minimized to reduce the size.

Third, a switch was applied to the front-end of the first-end LNA of the ultra-wideband receiver to secure two input ports capable of selectively receiving signals from two antenna paths so that signal incoming from a certain direction can be received more precisely. In addition, since signals coming in from other directions should be blocked to collect only those from a particular direction, single pole double throws (SPDT) were implemented in two layers so that the isolation between ports was at least $60 \mathrm{dBc}$. In this case, to obtain the targeted isolation characteristics, a wall was mechanically formed along the RF path so that the signals that go over to free spaces other than the transmission line can be blocked.

Fourth, since an EW system is composed of many devices such as antennas, a receiver, downconverter, signal processing unit, and power supply unit, which are connected to each other and installed in the aircraft, a directional coupler (D/C), which is not a switch part is designed and incorporated so that the built-in test (BIT) signals necessary to identify the malfunctioning device after installation can be injected into the front-end of the LNA. This enables the system to check itself for minimizing the deterioration of noise factor characteristics by reducing the losses in RF lines.

\section{II . ULTRA-WIDEBAND RECEIVER STRUCTURE}

In an ultra-wideband receiver, the very weak signals received from the antenna are low-noise amplified in the LNA and transmitted through a high pass filter (HPF) to suppress spurious signals other than the signals to be received and an image reject filter to suppress image signals. These signals are then applied to the frequency mixer and mixed with LO signals so that their frequencies are converted into IF. These resulting signals are transmitted through various kinds of filters, amplifiers, linearizers, and temperature compensation circuits, and finally applied to the signal processing unit. In this case, since the bands of the $\mathrm{RF}$ frequencies and the IF frequencies input to the receiver are very wide, the characteristics such as gain flatness, noise factor, and dynamic ranges deteriorate, and since a large number of components are integrated in the receiver and connected to each other, performance deteriorations frequently occur due to the occurrence of mismatch between the parts.

\section{RF Section}

Super heterodyne receivers (SHRs) use a time multiplexing scheme and are divided into narrow band (NB) SHRs and large band (LB) SHRs according to frequency bands. NB SHRs are used in ELINT systems to receive radiation signals in the surroundings without interference from other signals and LB SHRs are used in detecting or monitoring threatening emitters in high-density pulse environments. Advantages of SHRs include higher sensitivity than crystal video receivers, ability to detect minimum discernable signals from receiver inputs, easy removal of image signals as the image frequencies are far away from each other, and easy suppression of spurious waves [10]. Fig. 1 shows the block diagram of an SHR.

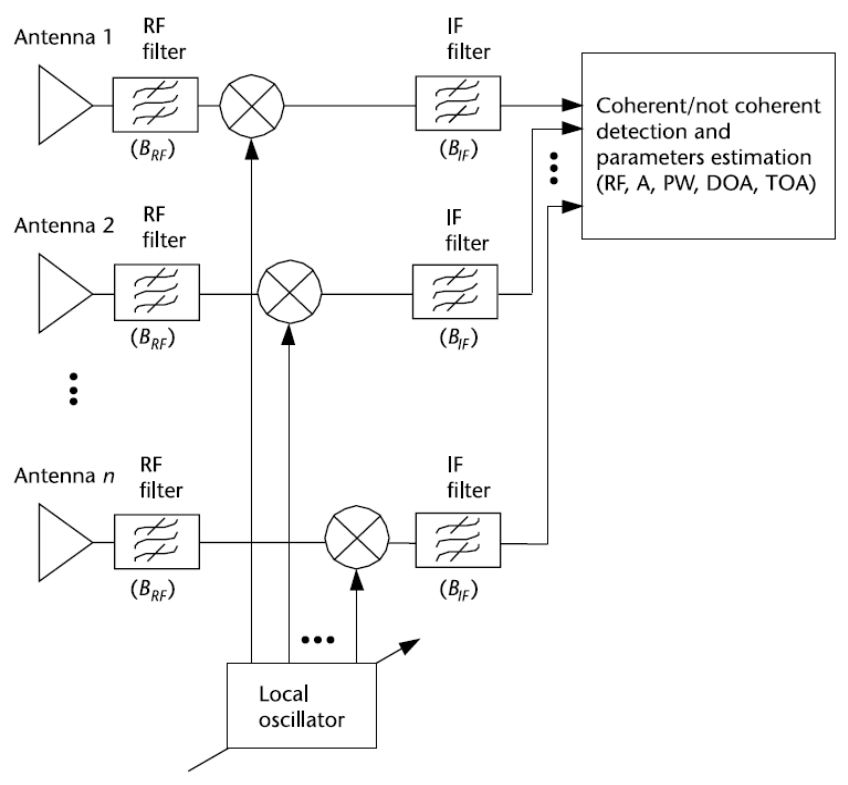

Fig. 1. Typical block diagram of a super heterodyne receiver. 
An SHR with excellent sensitivity and dynamic range characteristics, which are the main characteristics required for the millimeter-wave wideband receiver of EW equipment, was selected.

\section{LO Section}

LOs are circuits necessary to convert the frequency of the wideband receiver. The circuit and mechanical structure of the LO unit determine the performance of the entire receiver because the LO signals not only determine the phase noise characteristics of the entire receiver path, but the leakage signals and spurious signals (external reference signals, multiplied signals, mixed signal, etc.) included in the LO signals also affect the performance of the entire system through the frequency mixer [11]. The comb generator structure with excellent phase noise characteristics and little difficulty in the implementation of the circuit was selected. The harmonic component of input signals is output, while the input signals pass through the step recovery diode (SRD).

The harmonic appears as integer multiples of the input frequency at the output, and band pass filters are used to obtain the desired frequencies [12].

The phase noise of the $N$-multiplied frequency from external reference will be deteriorated by as much as the multiplying factor as per Eq. (1) below.

$$
\text { Phase Noise }=20 \operatorname{LOG} 10(N)
$$

Table 1. Analyzed values of the phase noise $(\mathrm{PN})$ for the comb generator method $(\mathrm{dBc} / \mathrm{Hz})$

\begin{tabular}{lccccc}
\hline & \multicolumn{5}{c}{ Offset frequency } \\
\cline { 2 - 6 } & 10 & 100 & 1 & 10 & 100 \\
& $\mathrm{~Hz}$ & $\mathrm{~Hz}$ & $\mathrm{kHz}$ & $\mathrm{kHz}$ & $\mathrm{kHz}$ \\
\hline External ref. PN & -70 & -100 & -130 & -140 & -155 \\
PN after multi- & -49 & -79 & -109 & -119 & -134 \\
plier (x11) & & & & & \\
\hline
\end{tabular}

Fig. 2 shows the block diagram of a comb generator. In particular, it is very important to design the signal generator so that it is not sensitive to the changes in ambient conditions its operation is very sensitive to external factors such as temperatures and vibrations [13]. Therefore, the above-mentioned factors should be considered when designing common sources.

Table 1 showed the analyzed values of the final phase noise of the comb generator method, which were $-109 \mathrm{dBc} / \mathrm{Hz}$ at $1 \mathrm{kHz}$ offset and $-119 \mathrm{dBc} / \mathrm{Hz}$ at $10 \mathrm{kHz}$ offset.

\section{DESIGN AND SIMULATION}

As shown in Fig. 3, the input frequency band was divided into Band A and Band B such that the secondary harmonic component does not exist in the band at the frequency mixer input terminal, thereby enabling the advance removal of mixed components in the band that may occur after down-conversion.

To compensate for such problems, the frequency conversion in the ultra-wideband receiver proposed in this paper was designed to minimize the complexity of the LO unit circuit and to output the LO frequency as a fixed frequency instead of variable frequencies to prevent the deterioration of the characteristics of phase noises and to enable spurious signal suppression to obtain an advantageous frequency spectra as the LO frequency is a single frequency. In addition, individual parts are connected with each other for fabrication and tests using microstrip lines or short RF cables and to reduce the mismatch in this case, additional tuning is also necessary.

\section{RF Section}

Fig. 4 shows the block diagram of the RF unit of the ultrawideband receiver designed in this study, with the two input ports of the receiver indicated using a dotted line on the upper part. The lower part indicated with a blue dotted line shows the block diagram of an LO circuit designed in this study, for which

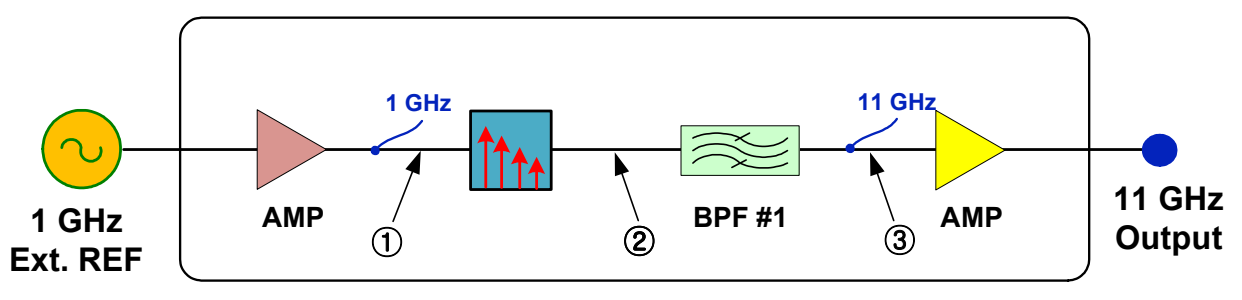

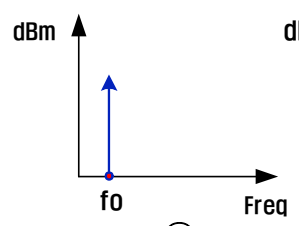

(1)

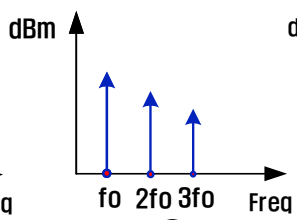

(2)

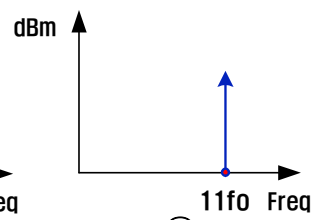

(3)

Fig. 2. Block diagram of a general comb generator method. 


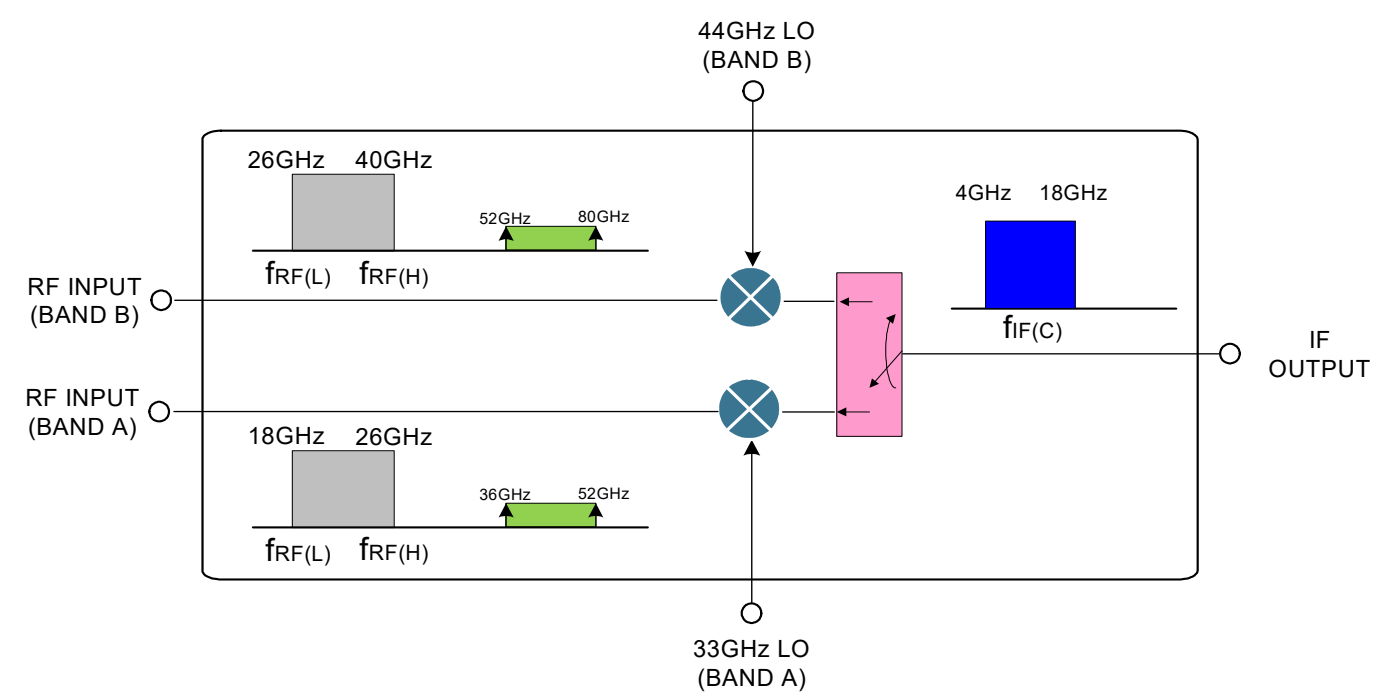

Fig. 3. Block diagram of the mixed signals in the IF band for $18-40 \mathrm{GHz}$ ultra-wideband receiver.

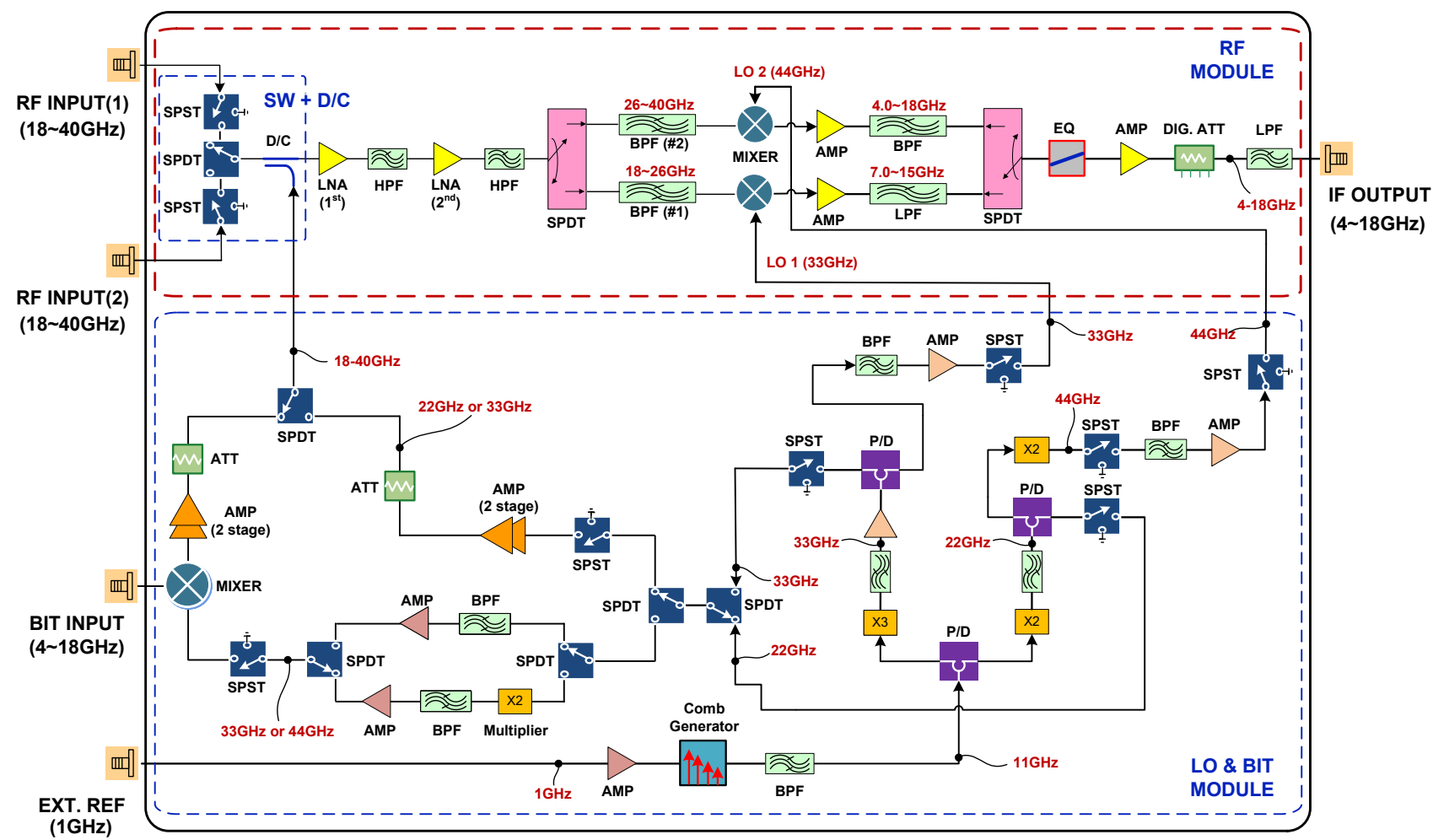

Fig. 4. Block diagram of RF unit of wideband down converter.

only the $1-\mathrm{GHz}$ external reference frequency was supplied, and the LO frequency and BIT signals were internally implemented.

\subsection{Noise figure analysis}

The designed first-end LNA is installed at the rear end of the SPST, SPDT, and D/C, as it should structurally secure the two input ports to satisfy the future requirements of EW systems. As the frequency increased, the noise factor of the LNA device was $3.5 \mathrm{~dB}$, indicating poor characteristics and if the deteriora- tion of $4.80 \mathrm{~dB}$ due to the loss at the front-end is added, the noise figure until the first-end of the LNA can be expected to be approximately $8.30 \mathrm{~dB}$.

\subsection{Spurious analysis}

As can be seen in Fig. 5, unwanted frequencies outside the band intrude into the signals received by the antenna. In this case, after passing the signals through the LNA, an HPF is used to suppress spurious signals in low frequency bands at point $\mathrm{B}$. 


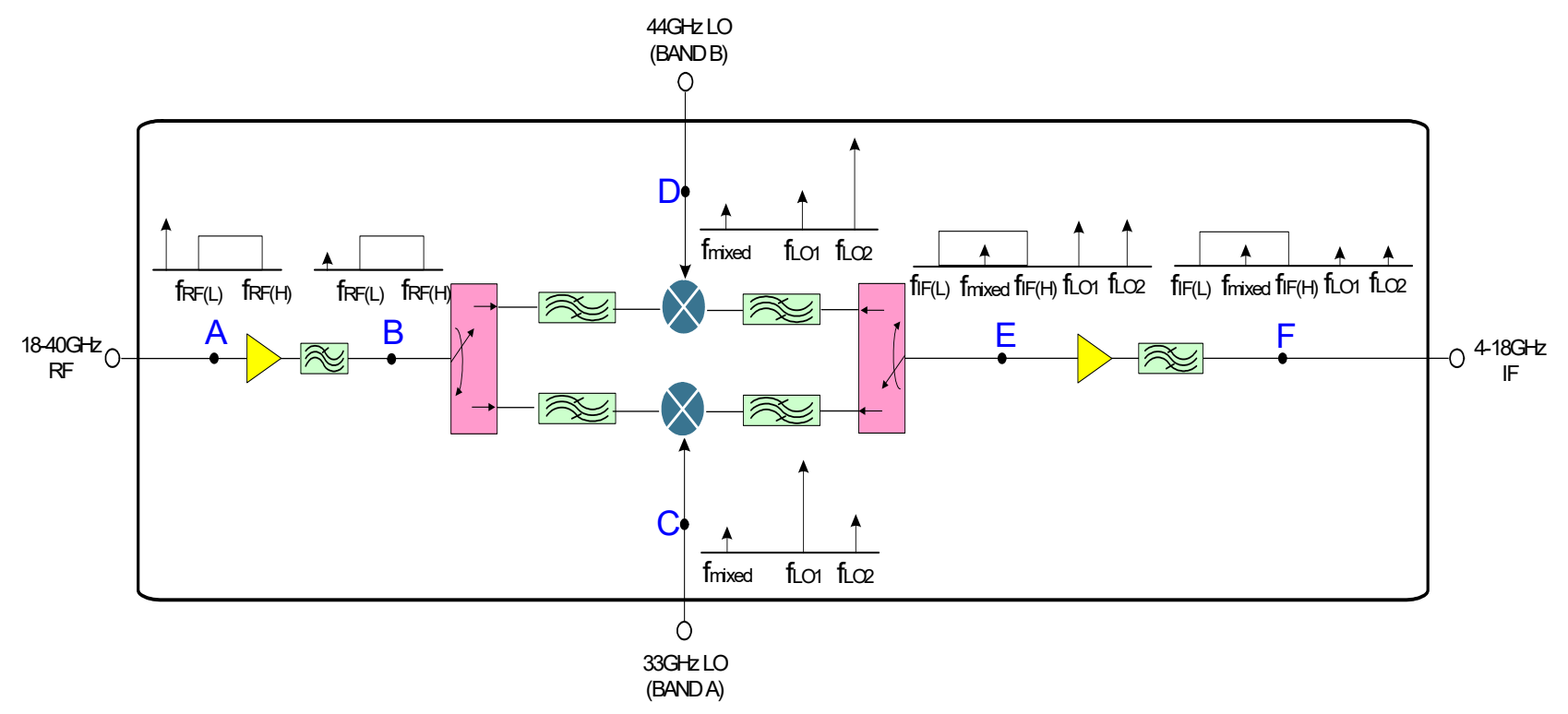

Fig. 5. Block diagram of frequency spectrum of the entire RF.

Point $\mathrm{C}(33 \mathrm{GHz})$ and point $\mathrm{D}(44 \mathrm{GHz})$ are the spectra of LO signals for frequency conversion, and it can be seen that spurious signals other than the desired signals are generated by many components such as the multiplier and filters after phase synchronization with the $1-\mathrm{GHz}$ external reference frequency, until the signals obtain the final output frequency. When these frequencies have gone through the mixer, as with point $\mathrm{E}$, in addition to the desired IF frequency band, LO signals $\left(f_{L O 1}, f_{L O 2}\right)$ and $11 \mathrm{GHz}$ signals, which are mixed signals of two LO frequencies become to exist in the band. At point $\mathrm{F}$ where the signals have passed through the low pass filter, LO signals (33 $\mathrm{GHz}, 44 \mathrm{GHz}$ ) can be removed.

\subsection{Feed thru design}

In general, the impedance is well matched in plane structures, as the direction of progression of the electric field is the same as the transmission line. In the case of vertical structures, impedance mismatching is not common on the side where the core of the feed thru is connected as air spaces can be made mechanically; however, impedance matching through the adjustment of surroundings via holes and tuning is necessary on the opposite side due to weak $50 \Omega$. In addition, the assembly process is more important than the design. To fix the feed thru to the instrument, an epoxy material is used. The epoxy is hardened for approximately 15 minutes at high temperatures of around $120^{\circ} \mathrm{C}$. In this case, if the hardening conditions are not satisfied or fine cracks occur, losses rapidly grow in the ultra-high-frequency band so that the LO signal power supplied to the mixer is reduced [14].

Fig. 6 shows the side view of the structure to which the feed thru is connected. Fig. 7 is the result of simulation of feed thru

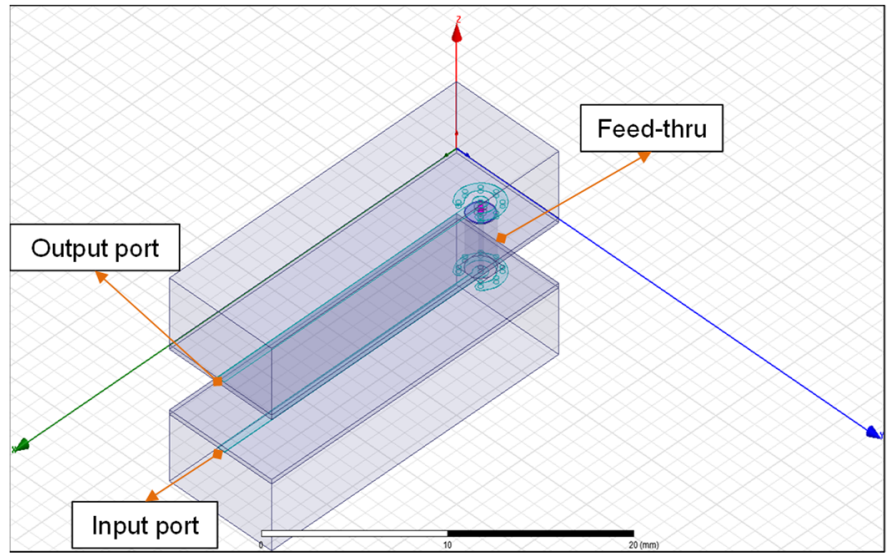

Fig. 6. Vertical structure simulation (3D) using the feed thru.

in a vertical structure, which shows the $33 \mathrm{GHz}$ signal applied from the LO module to the mixer of the RF module. The insertion loss was shown to be not larger than approximately -1.1 $\mathrm{dB}$ and the reflection was shown to be not larger than $-19 \mathrm{~dB}$.

\section{LO Section}

Fig. 4 shows a block diagram of an LO circuit designed in this paper, of which only the $1 \mathrm{GHz}$ external reference frequency was licensed, and the LO frequency and external BIT signals were internally implemented as the part marked with a red dotted line (square box on the lower part).

\subsection{Comb generator design}

To synthesize the LO signals ( $33 \mathrm{GHz}, 44 \mathrm{GHz}$ ) using the external reference signal of $1 \mathrm{GHz}$, the $11 \mathrm{GHz}$ frequency should first be designed using the harmonic component of the comb generator. A step recovery diode (Aeroflex Inc., Plainview, 


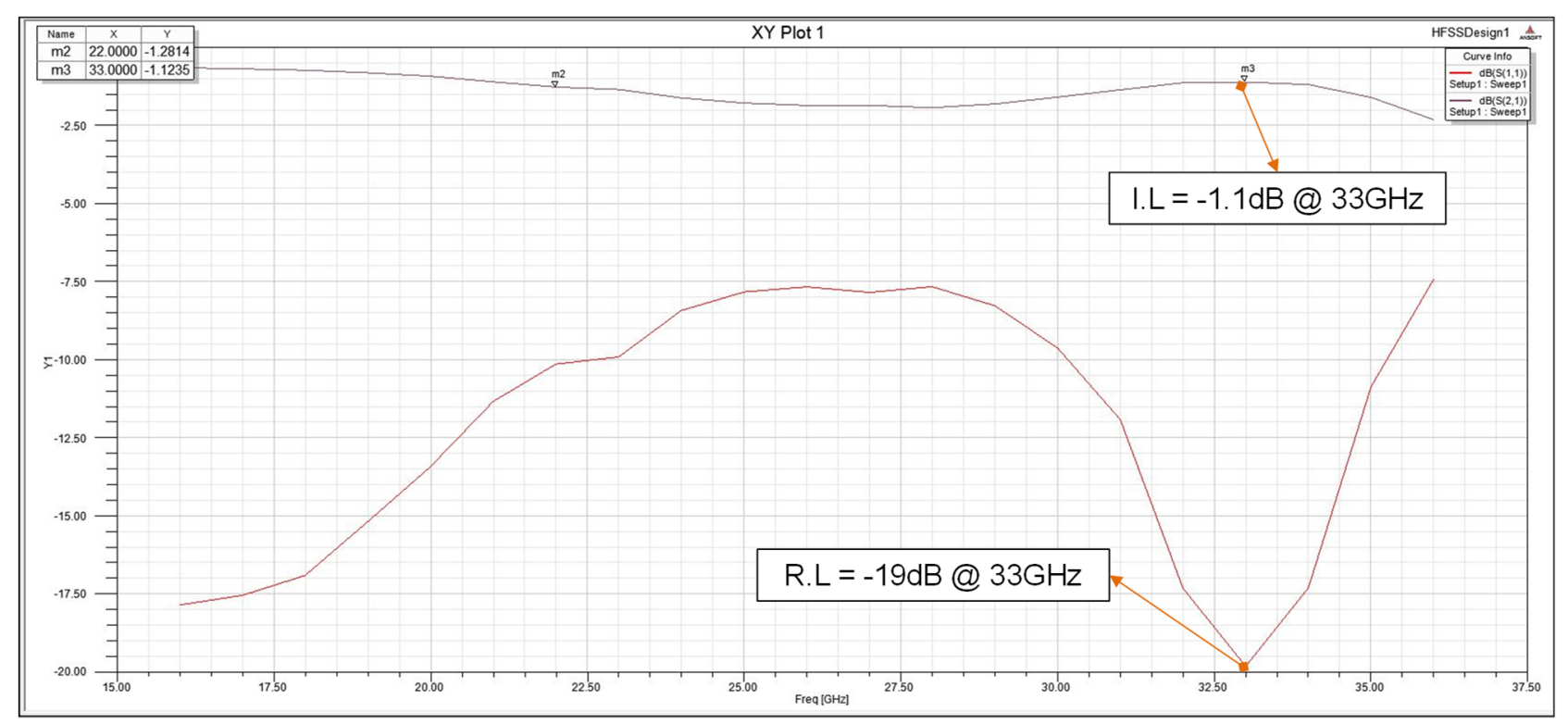

Fig. 7. Result of vertical structure simulation using the feed thru.

NY, USA) was used, while matching was induced using an inductor and capacitor in order to match the low impedance of the SRD input with $50 \Omega$. A TQP3M9009 amplifier (Triquint, Hillsboro, OR, USA) was used to amplify the low reference signal input power $(0 \mathrm{dBm})$ while driving the SRD. Figs. 8 and 9 show the design and result of the simulation to generate the $11 \mathrm{GHz}$ frequency.

\subsection{LO leakage analysis}

In Fig. 10, point $A$ is the external reference frequency of 1 $\mathrm{GHz}$ provided by the EW system, and at point $\mathrm{B}$, the frequency that remains after passing through the comb generator circuit and band pass filter, which is $11 \mathrm{GHz}$ among the harmonic frequencies that are integer multiple of $1 \mathrm{GHz}$, can be seen. In this case, suppressing harmonic components other than the 11

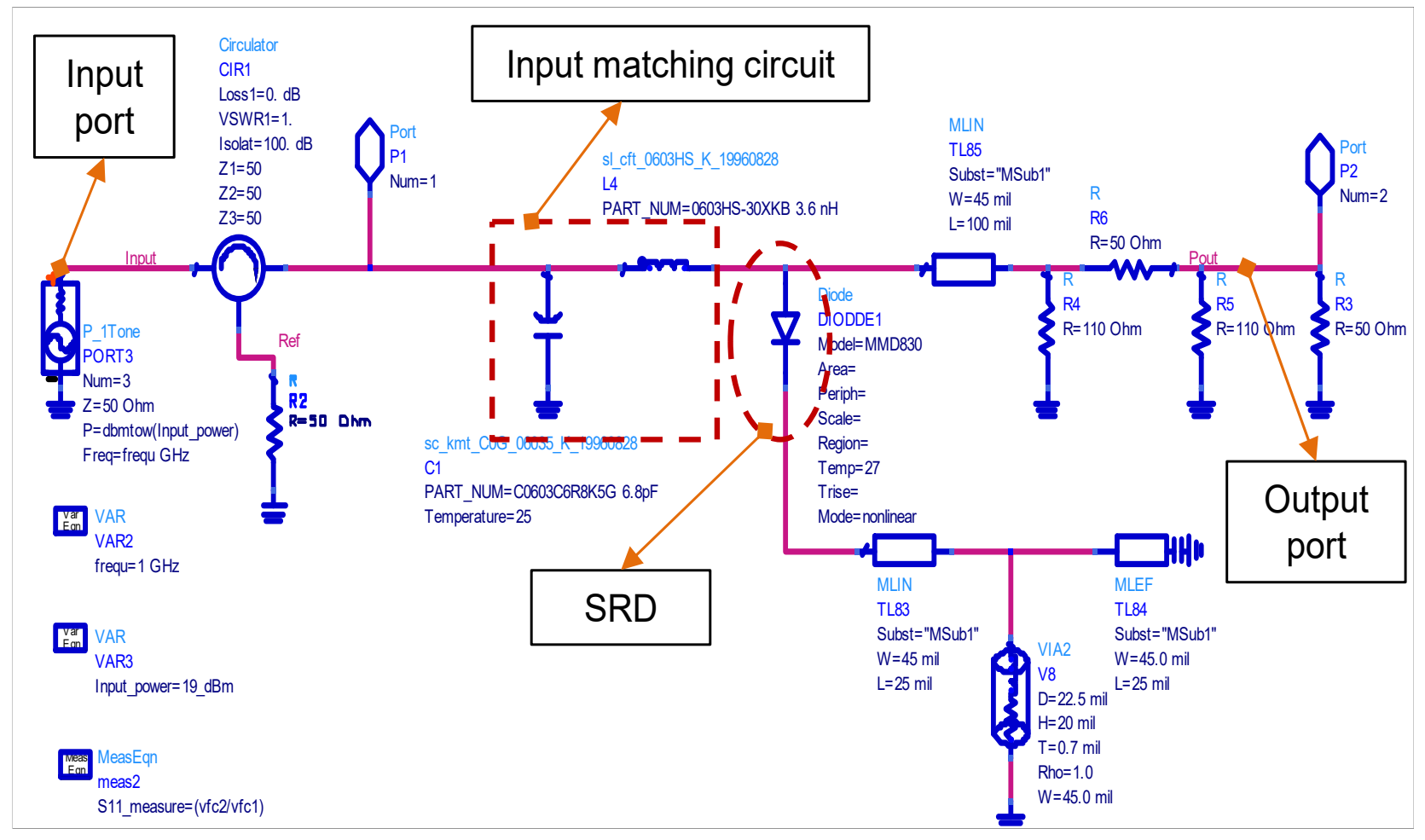

Fig. 8. Circuit of simulation for the comb generator circuit. 


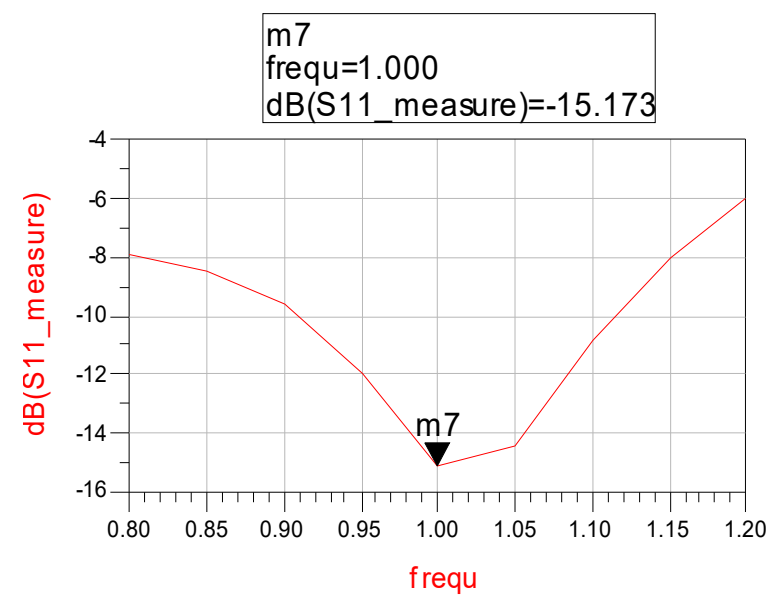

(a)

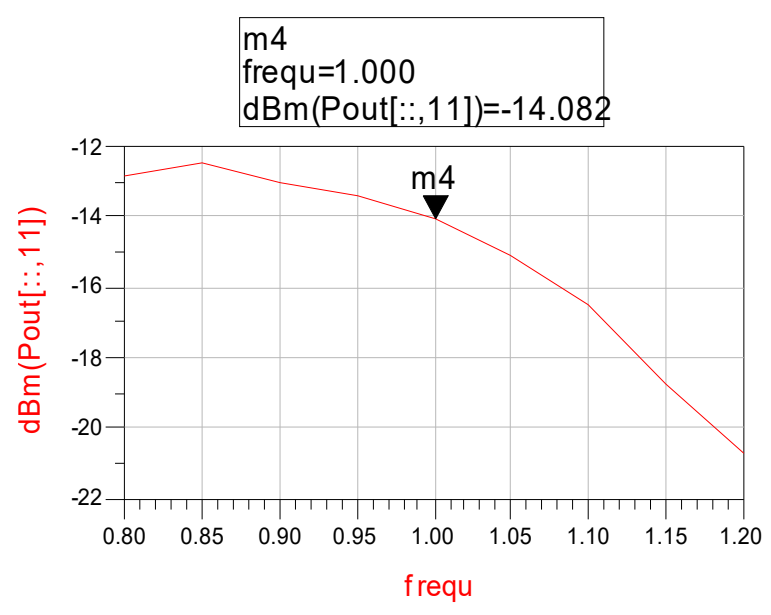

(b)

Fig. 9. Result of the simulation for the comb generator circuit. (a) Reflection coefficient of $11 \mathrm{GHz}$ harmonic signal and (b) output power of the 11th order harmonic signal.

$\mathrm{GHz}$ signal is very important. Point $\mathrm{C}(33 \mathrm{GHz})$ and point $\mathrm{D}$ $(44 \mathrm{GHz})$ are the LO signal spectra for frequency conversion, generated using many components such as the multiplier, filter, and amplifier.

\subsection{HPF design}

The structure of the 13-pole HPF in the microstrip structure is shown in Fig. 11. The designed HPF PCB information shows that the dielectric constant of the alumina substrate is 9.8 and the height is $0.254 \mathrm{~mm}$.

Fig. 12 shows the design, in which the reflection coefficient is not higher than $-12.7 \mathrm{~dB}$ and the insertion loss is about $-0.6 \mathrm{~dB}$ at an $\mathrm{LO}$ frequency of $33 \mathrm{GHz}$. In addition, the rejection value at $22 \mathrm{GHz}$ is about $34 \mathrm{dBc}$, which was designed so that 22

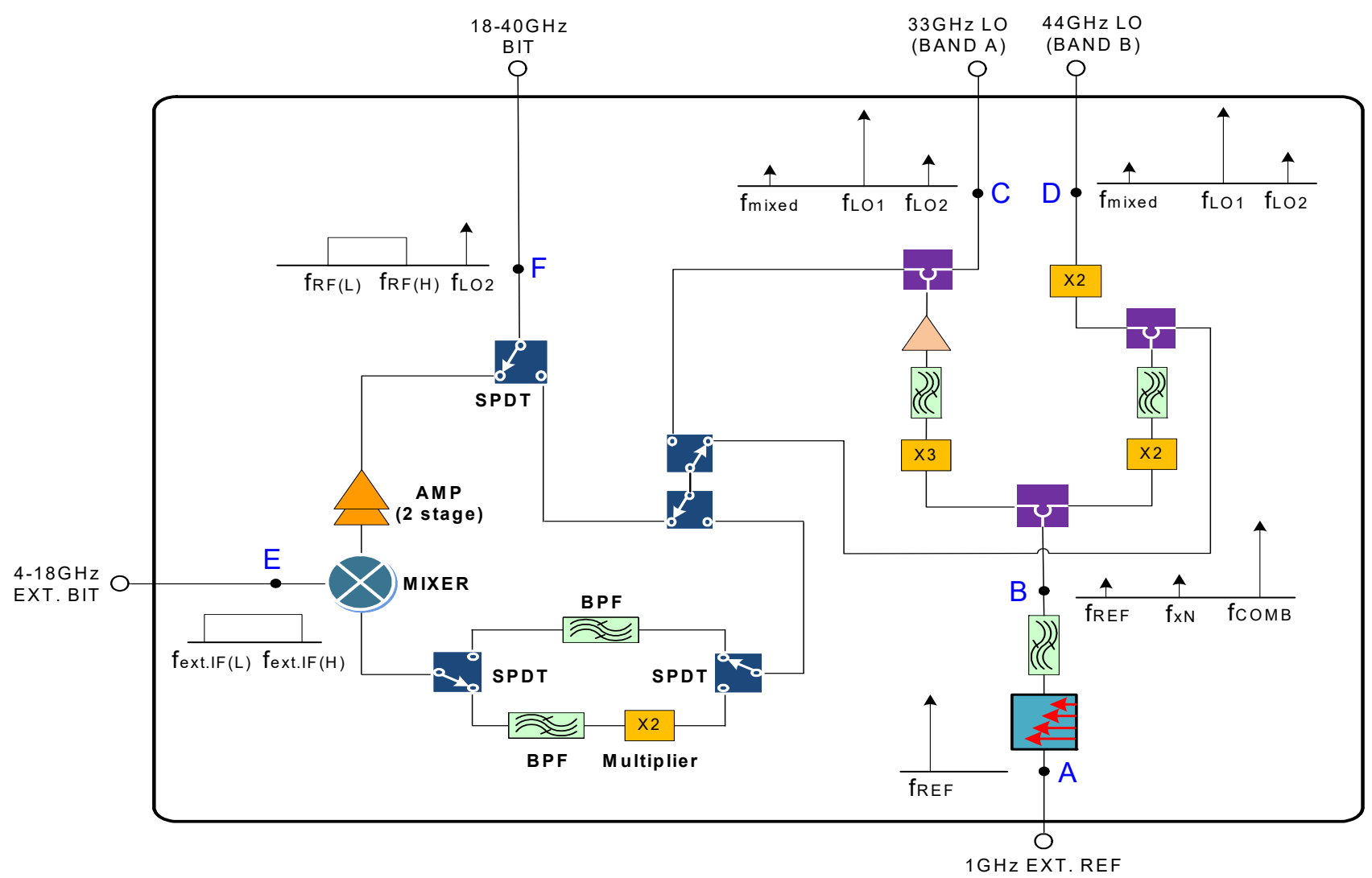

Fig. 10. Block diagram of frequency spectrum for the LO circuit. 


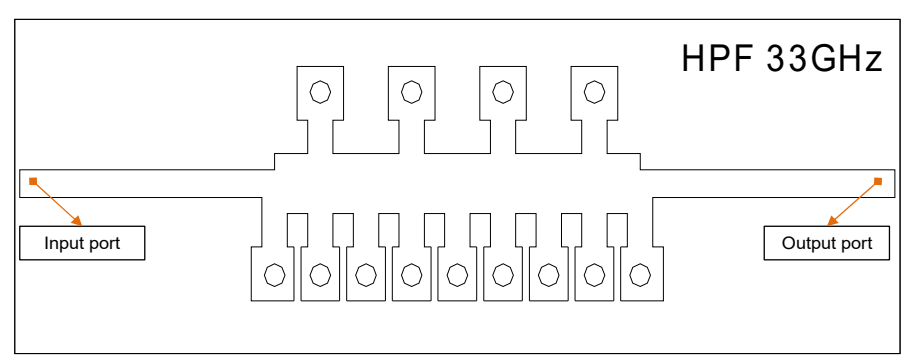

Fig. 11. HPF layout.

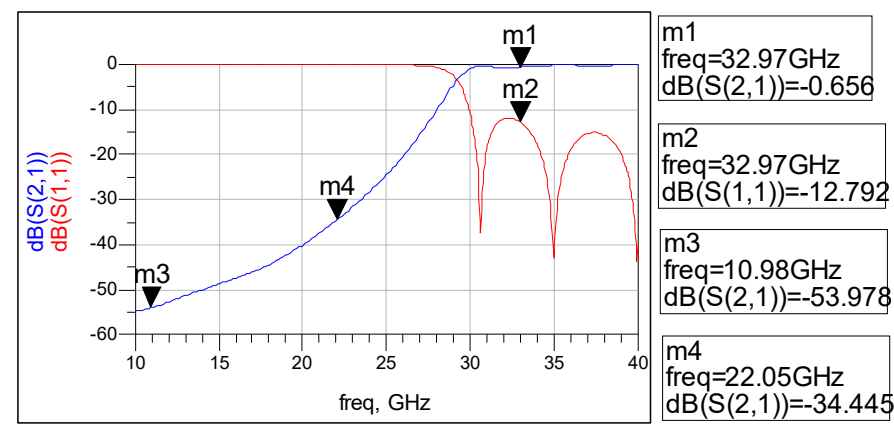

Fig. 12. Results of HPF (33 GHz) simulation.

$\mathrm{GHz}$ spurious signals can be sufficiently suppressed when they exist in the $33 \mathrm{GHz}$ path.

\section{FABRICATION AND MEASUREMENT}

To design the wideband receiver module having two RF input ports (18-40 GHz), one IF output port (4-18 GHz), a 1$\mathrm{GHz}$ reference port to match the phase of the internal signal source with that of external reference signal sources, and an external BIT input port $(4-18 \mathrm{GHz})$, the RF area and the LO were first designed to be mechanically separated. Sub-module units were assembled in each area and each sub-module was assembled using bare MMIC and Rogers RT/duroid 5880 (thickness, $0.127 \mathrm{~mm}$ ) PCB to achieve downsizing and weight reduction. When connecting the sub-module units with each other, adaptors such as connectors or bullets were used to minimize losses in ultra-high-frequency bands and the cover was made using an epoxy material so that it can be attached to the body of the instrument. From assembly point of view, RF modules in the very high-frequency $40 \mathrm{GHz}$ range must be assembled using bare MMICs instead of packaged components due to impedance mismatch. After attaching MMIC parts and high-frequency PCB to the mechanical housing, 1 mil wire bonding is used to connect parts and transmission line. This assembly process is called chip-and-wire process.

The entire circuit was implemented, reflecting the values of the sub-unit circuits constituting the RF module verified through simulations. Fig. 13 shows a photo of the actually fabricated module based on the drawing of the finally designed RF

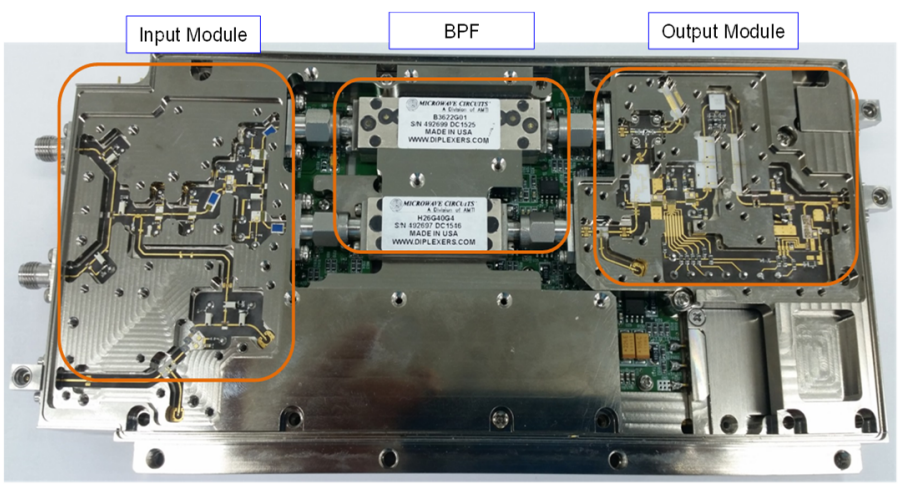

Fig. 13. Photo of the fabricated RF module.

artwork in which the RF input module, the band pass filter, and the IF output module are combined.

The entire circuit was implemented reflecting the values of the sub-unit circuits constituting the LO module verified through simulations. Fig. 14 shows a photo of an actually fabricated LO module based on the design in which a 1-GHz REF module, an LO module, and a BIT module are combined.

\section{Gain Measurement Result}

Fig. 15 shows gain graphs for Band A (input frequency, 18$26 \mathrm{GHz}$ ) and Band B (input frequency, 26-40 GHz). Fig. 15(a) shows the results for input port 1 in which the gain was between $22.22 \mathrm{~dB}$ at the minimum and $28.55 \mathrm{~dB}$ at the maximum with a gain flatness of approximately $6.33 \mathrm{~dB}$. Fig. 15(b) shows the results for input port 1 in which the gain was between $21.9 \mathrm{~dB}$ and $26.1 \mathrm{~dB}$ with an approximate gain flatness $4.1 \mathrm{~dB}$.

\section{NF Measurement Result}

The $\mathrm{RF}$ input frequencies of $18-40 \mathrm{GHz}$ are values converted with IF frequencies of $4-18 \mathrm{GHz}$ by 33 and $44 \mathrm{GHz} \mathrm{LO}$ signals. As shown in Fig. 16, the noise factors are the results of measurement of the noise factors for Band $\mathrm{A}$ (input frequency, 18-26 GHz) and Band B (input frequency, 26-40 GHz) and showed values ranging from $7.92 \mathrm{~dB}$ at Band $\mathrm{A}$ to $8.58 \mathrm{~dB}$ at

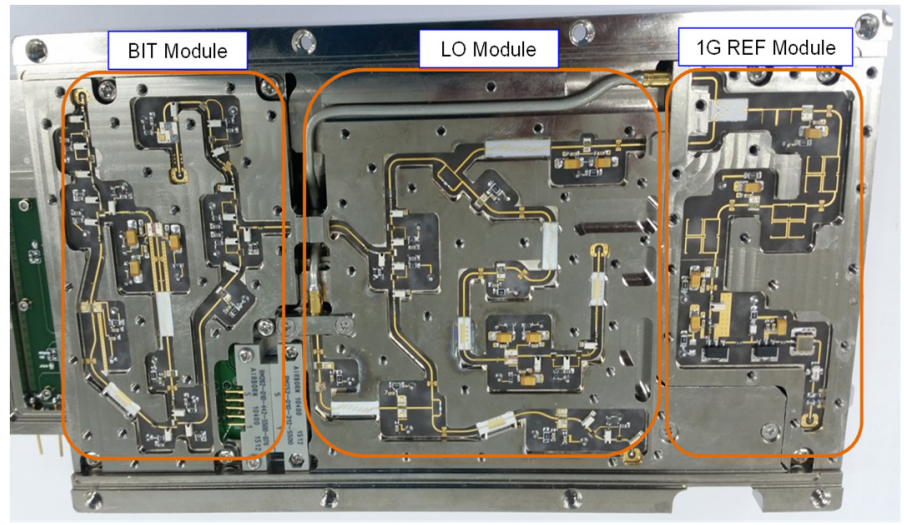

Fig. 14. Photo of the fabricated LO module. 


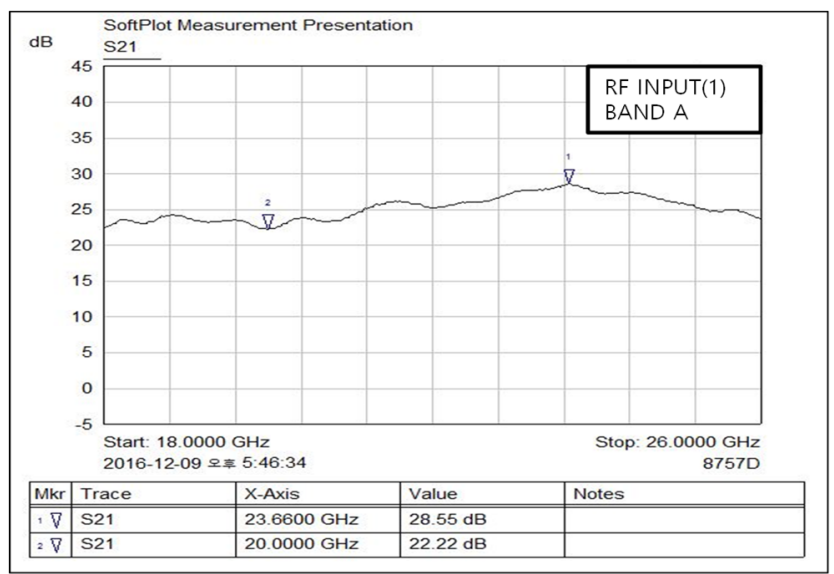

(a)

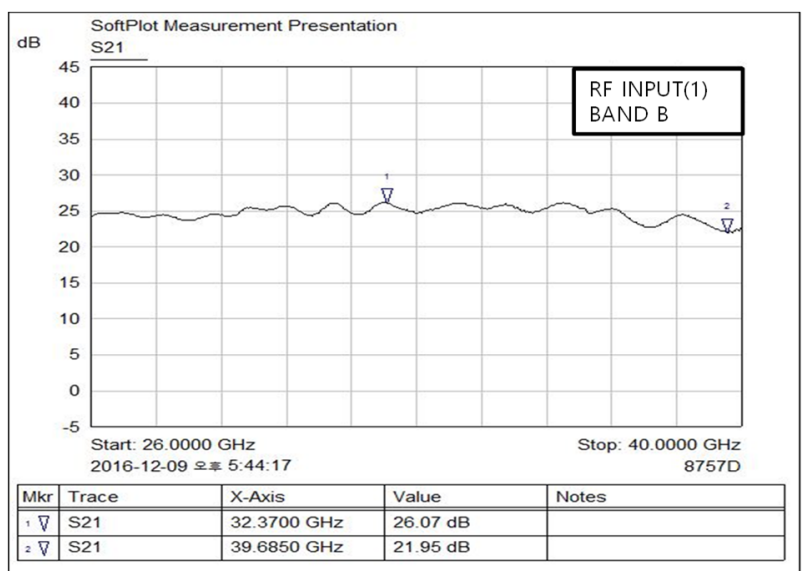

(b)

Fig. 15. Results of measurement of gains by band of antenna paths. Gains of Band A (a) and Band B (b) for RF input (1) path.

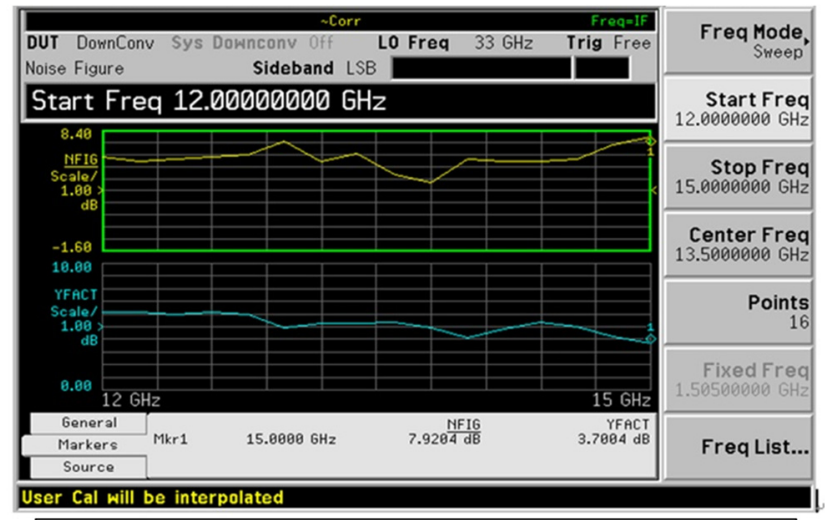

Band A

RF Input $(18-21 \mathrm{GHz}) /$ RF Output $(12-15 \mathrm{GHz})=7.92 \mathrm{~dB} \max$.

(a)

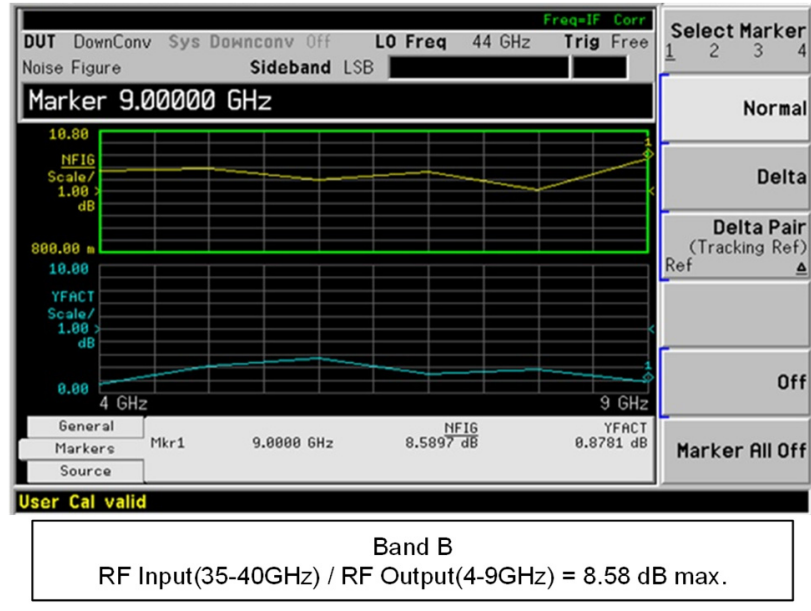

(b)

Fig. 16. Results of measurement of gains by bands of antenna paths. Noise figures of Band A (a) and Band B (b).

Band $B$ within each band.

\section{Second Harmonic Measurement Result}

The second harmonic component at the IF output port, which is different from the intermodulation distortion signals, is a characteristic output due to the active component (amplifier) used inside the wideband receiver, and affects the sensitivity depending on the suppressed quantity in the signal processing area because it cannot be suppressed even with filters within the band. After inputting $-17 \mathrm{dBm}$ (almost the maximum input power) into the input port, the harmonic component was measured at the IF output port using a spectrum analyzer.

Fig. 17 shows the result of measurement of the harmonic. Fig. 17(a) is the result of measurement at the $15 \mathrm{GHz}$ IF output of band $\mathrm{A}(\mathrm{LO}, 33 \mathrm{GHz})$ and the suppression characteristic obtained through the measurement was approximately $30 \mathrm{dBc}$. Fig. $17(\mathrm{~b})$ is the result of measurement at the $4 \mathrm{GHz}$ IF output of band $\mathrm{B}(\mathrm{LO}, 44 \mathrm{GHz})$ and the suppression characteristic ob- tained through the measurement was approximately $36 \mathrm{dBc}$.

Fig. 18 shows the measured value of the 33 and $44 \mathrm{GHz} \mathrm{LO}$ leakage power, which showed suppression characteristics between -97.3 and $-90.1 \mathrm{dBm}$, approximately. Since the LO leakage signals do not exceed $-80 \mathrm{dBm}$, which is lower than the threshold for signal processing, it can be expected that the LO leakage signals have no effect.

\section{CONCLUSION}

In this paper, we designed and fabricated a small, lightweight, reliable, and stable receiver with low noise figure, flat gain characteristics, and low noise characteristics by applying the chipand-wire process for the assembly and operation of a bare MMIC device. In order to compensate for the mismatch between many components used in the receiver, an amplifier, mixer, multiplier, and filter suitable for the wideband frequency characteristics were designed and applied to the receiver. To 


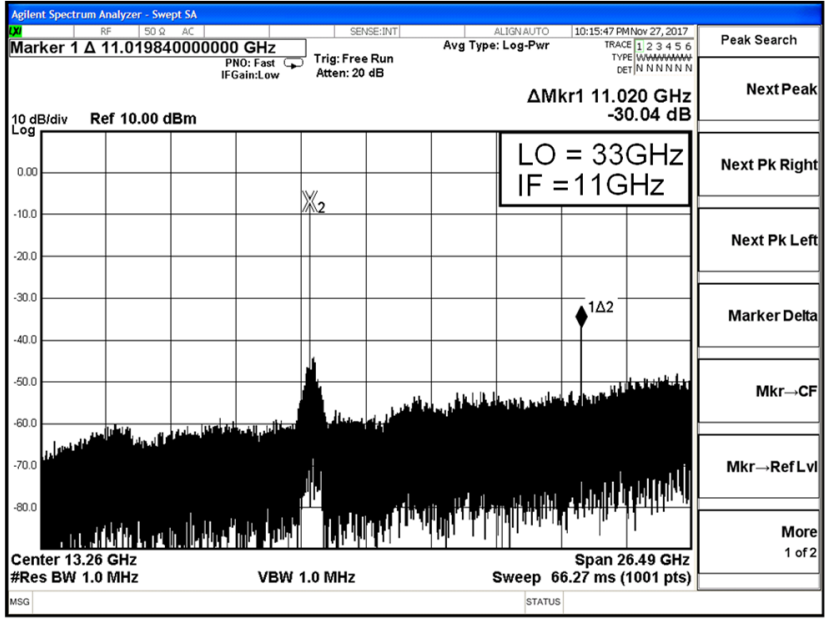

(a)

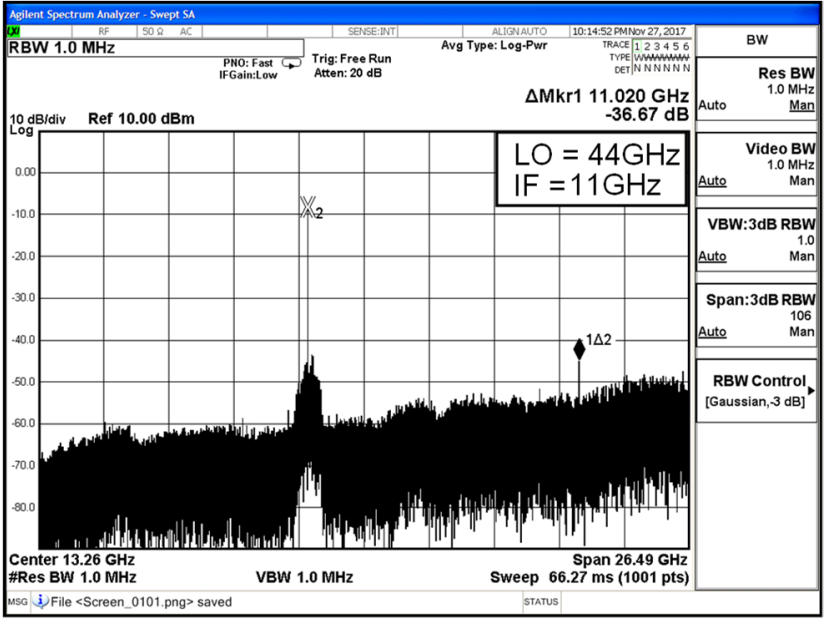

(b)

Fig. 17. Results of measurement for secondary harmonics at IF frequency of $11 \mathrm{GHz:}$ (a) Band A and (b) Band B.

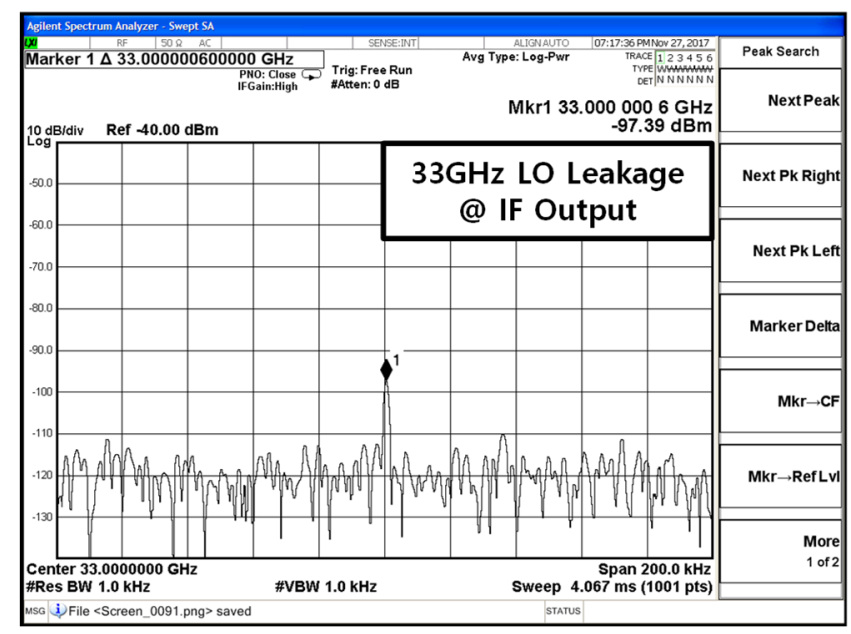

(a)

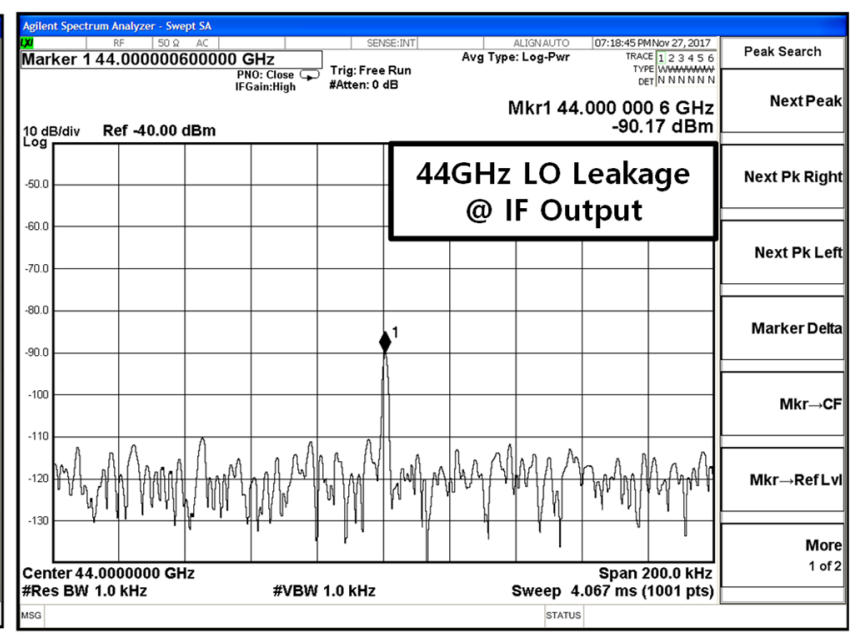

(b)

Fig. 18. Results of measurement of 33 and $44 \mathrm{GHz}$ spurious signals @ IF: (a) Band A and (b) Band B.

improve the low frequency and narrow bandwidth of existing products, mathematical modeling of the wideband receiver was performed and based on this, spurious signals generated from complex LO signals were designed so as not to affect the RF path.

In the ultra-wideband receiver, the gain was between $22.2 \mathrm{~dB}$ and $28.5 \mathrm{~dB}$ at Band $\mathrm{A}$ (input frequency, 18-26 GHz) with a flatness of approximately $6.33 \mathrm{~dB}$ while at Band B (input frequency, 26-40 GHz), the gain was between $21.9 \mathrm{~dB}$ and 26.0 $\mathrm{dB}$ with a flatness of approximately $4.1 \mathrm{~dB}$. The measured value of noise figure at Band A was $7.92 \mathrm{~dB}$ and at Band B was 8.58 $\mathrm{dB}$ with a maximum value. The leakage signal of the $\mathrm{LO}$ was -97.3 and $-90 \mathrm{dBm}$ at the 33 and $44 \mathrm{GHz}$ paths, respectively. Measurement was conducted at the $15 \mathrm{GHz}$ IF output of band A $(\mathrm{LO}, 33 \mathrm{GHz})$ and the suppression characteristic obtained through the measurement was approximately $30 \mathrm{dBc}$.

The 18-40 GHz band ultra-wideband receiver proposed in this paper satisfied the strict performance requirements (wide bandwidth, flat gains, low noise factor, wide dynamic range, and low spurious signals) necessary for EW systems and is suitable for application to the front-end module of an EW that must receive high-frequency band signals.

\section{REFERENCES}

[1] B. S. Kim, "The present situation and development trend of EW equipment technology for installation in aircraft," 2011.

[2] W. B. Sullivan, "Receivers reach new thresholds," Journal of Electronic Defense, vol. 17, no. 12, pp. 52-52, 1994.

[3] R. Sarath and C. Viswanadham, "Receiver processor for controlling ESM systems," International Journal of Engineering Trends and Technology, vol. 4, no. 9, pp. 3805-3808, 2013.

[4] S. T. Winnall and D. B. Hunter, "A fibre Bragg grating based scanning receiver for electronic warfare applications," in Proceedings of 2001 International Topical Meeting on Mi- 
crowave Photonics, Long Beach, CA, 2002, pp. 211-214.

[5] D. E. Allen, "Channelised receiver: a viable solution for EW and ESM systems," IEE Proceedings F (Communications, Radar and Signal Processing), vol. 129, no. 3, pp. 172-179, 1982.

[6] R. T. Logan and R. D. Bynum, "Millimeter wave optical link/frequency converter system," Tracor Aerospace Electronic Systems Inc., Lansdale, PA, Report No. AFRL-SNRS-TR-1998-160, 1998.

[7] L. M. Devlin, G. A. Pearson, A. W. Dearn, P. D. L. Beasley, and G. D. Morgan, "A 2-18 GHz ESM receiver front-end," in Proceedings of 32nd European Microwave Conference, Milan, Italy, 2002, pp. 1-4.

[8] M. S. Rao, "EMI/EMC effects on EW receiver systems of military aircraft," in Proceedings of the 10th International Conference on Electromagnetic Interference E Compatibility, Bangalore, India, 2008, pp. 63-67.

[9] W. R. Brinlee, A. M. Pavio, C. L. Goldsmith, and W. J. Thompson, "A monolithic multifunction EW broadband receiver converter," in Proceedings of the 15th Annual Gallium

Yuseok Jeon

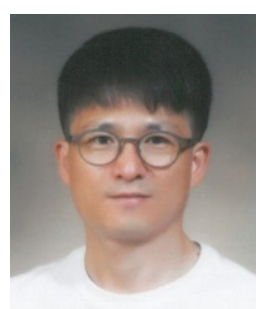

obtained his B.S. in electronic communication engineering from Cheongju National University, Cheongju, Korea, in 2000, his M.S. in electronic engineering from Chungbuk National University, Cheongju, Korea, in 2004, and his Ph.D. in electronic and electric engineering from Dankook University, Yongin, Korea, in 2018. He worked at Broadern Inc., Hwaseong, Korea, from 1999 to 2018. His research interests include the development of transceivers, receivers, and synthesizers using chip-and-wire processes for EW and radar system applications.
Arsenide Integrated Circuit (GaAs IC) Symposium, San Jose, CA, 1993, pp. 207-210.

[10] S. R. Bullock, Transceiver and System Design for Digital Communications. Atlanta, GA: Noble Publishing, 1995.

[11] H. Fudem, G. Dietz, R. Haubenstricker, R. Hargis, and S. Moghe, "A high performance 6 to $18 \mathrm{GHz}$ MMIC converter chip for EW systems," in Proceedings of the 12th Annual Gallium Arsenide Integrated Circuit (GaAs IC) Symposium, New Orleans, LA, 1990, pp. 113-116.

[12] Picosecond Pulse Labs., "A new breed of comb generators featuring low phase noise and low input power," Microwave Journal, vol. 49, no. 5, pp. 278-280, 2006.

[13] Y. S. Jeon, B. C. Kim, and J. M. Jeong, "Phase locked Oscillator using a comb generator," KR Grant KR101255231B1, Apr. 2013.

[14] Thunderlinez, "Top ten soldering pitfalls and how to avoid them," [Online]. Available: https://climate.emerson.com/ documents/soldering-pitfalls-how-to-avoid-them-en-us-162 0698.pdf.

Sungil Bang

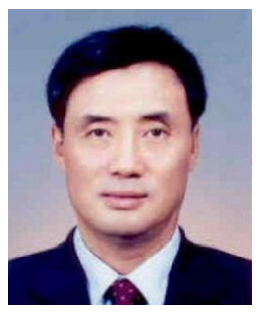

obtained his B.S. in electronic engineering from Dankook University, Seoul, Korea, in 1984, his M.S. in electronic engineering from Dankook University, Seoul, Korea, in 1986, and his Ph.D. in electronic engineering from Dankook University, Seoul, Korea, in 1992. From 1994 to 1997, he worked as the director of the research division at LC Tech Inc., Anyang, Korea. Since 1994, he has been a Professor of Electronic and Electric Engineering at Dankook University, Yongin, Korea. His research interests include RF Amp, UWB, OFDM, and RFID for telecommunication applications. 